\title{
La Vieille latine témoin du Prôto-Marc
}

Par

\section{Christian-B. Amphoux}

\author{
CNRS, Aix-Marseille Université
}

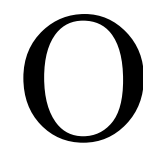

n doit à Jean-Claude Haelewyck l'édition scientifique la plus complète de la version latine de Marc d'avant la Vulgate, publiée dans la collection de la Vetus latina ${ }^{1}$, après l'editio princeps de la Bible latine d'avant la Vulgate par Pierre Sabatier (Reims, 1743) et la Vetus itala des évangiles, par Adolf Jülicher (Berlin, 1963-1976; pour Marc, 1970). Le texte latin de cette version a été établi sur la base de 18 manuscrits et du répertoire complet des citations patristiques, grâce au fichier de Beuron.

Il y a quarante ans, en avril 1982, en m'interrogeant sur la composition littéraire de l'évangile de Marc, j'ai découvert qu'une structure régulière unissait les quelque 40 épisodes disposés dans le même ordre dans les trois synoptiques, et j'en conclus alors qu'ils avaient une origine commune, distincte de celle(s) des autres épisodes. Mais qu'en était-il donc des autres ? La découverte inopinée de la lettre de Clément d'Alexandrie sur un évangile secret de Marc, publiée par son inventeur, Morton Smith, en $1973^{2}$ et traduite en français dans les Écrits apocryphes chrétiens par Jean-Daniel Kaestli ${ }^{3}$, vint m'apporter une réponse, en dépit de la réputation que cette lettre était un faux, peut-être l'œuvre de son inventeur. Si l'on isole

\footnotetext{
${ }^{1}$ Jean-Claude HAELEWyCK, Evangelium secundum Marcum, Vetus latina, Die reste der altlateinischen Bibel, vol. 17, Freiburg-im-Brisgau, Verlag Herder, 2013-2018, 10 fasc., 800 p.

${ }^{2}$ Morton SMIth, Clement of Alexandria and a Secret Gospel of Mark, Cambridge (Mass.), 1973, p. 448-449.

${ }^{3}$ Jean-Daniel KAESTLI, « Evangile secret de Marc », dans François BovON et Pierre GeOLTRAIN (dir.), Écrits apocryphes chrétiens, vol. 1, La Pléiade, Paris, Gallimard, 1997, p. 55-69.
} 
dans Marc les épisodes ordonnés des synoptiques et quatre autres qui en sont des annexes ${ }^{4}$, peut-être ajoutés dans un deuxième temps, il reste quelque 22 épisodes correspondant parfaitement à la description du livre de Marc attestée dans la lettre de Clément d'Alexandrie. À première vue, ces épisodes ne sont pas liés entre eux par l'histoire qu'ils racontent; mais le lien apparaît par un deuxième sens selon lequel ils s'organisent en trois séries, l'une principale et les autres secondaires, montrant le bien-fondé du témoignage de la lettre de Clément d'Alexandrie.

Le passage du sens apparent au deuxième sens se fait parfois sans difficulté ; mais il arrive qu'il soit masqué par une révision du texte qui a fait abandonner la variante primitive ; et nous allons voir que ces variantes donnant accès au deuxième sens sont généralement celles du Codex de Bèze (mais pas toujours), le plus souvent partagées avec la Vieille latine et par elle seulement. Notre plan sera le suivant : 1. La composition du Prôto-Marc ; 2. Les métaphores permettant le passage au deuxième sens ; 3 . Les accès au deuxième sens perdus par la Vieille latine ; 4. Les accès conservés par la Vieille latine.

\section{La composition du Prôto-Marc}

Le Prôto-Marc, envisagé par Schleiermacher ${ }^{5}$, a vainement été recherché au $19^{\mathrm{e}}$ siècle, et le $20^{\mathrm{e}}$ a finalement identifié tout Marc à la source narrative de Matthieu et Luc, attestée par Papias.

Le témoignage de Clément d'Alexandrie, inconnu au $19^{\mathrm{e}}$ siècle, distingue trois séries dans le livre de Marc: un premier écrit catéchétique, écrit du vivant de Pierre à Rome et comprenant des récits mettant en scène Jésus ; puis deux séries complémentaires, ajoutées à Alexandrie après la mort de Pierre, l'une narrative, reprenant des «mémoires » de Pierre et destinée à une formation de fin d'étude, et l'autre contenant des paroles de Jésus, réservée à un auditoire encore plus restreint de responsables de communautés ${ }^{6}$. Notre ensemble de 22 épisodes s'organise d'une manière comparable : (1) 12 épisodes qui ont un parallèle dans Luc forment une première série représentant la rédaction de Marc destinée à la catéchèse ; (2) 5 épisodes formant une même séquence narrative ayant un parallèle dans Matthieu, et pas dans Luc, correspondent au complément narratif issu de «mémoires » de Pierre et destiné à un

\footnotetext{
${ }^{4}$ Mc 6,17-29 (la mort de Jean) ; 10,1-12 (l'union et la désunion) ; 14,22-25 (le dernier repas) ; et 14,55-65 (le jugement du sanhédrin). De plus, la Finale longue $(16,9-20)$ ne fait pas originellement partie de Marc.

${ }^{5}$ Friedrich SCHLEIERMACHER, « Über die Zeugnisse des Papias von unsern beiden ersten Evangelien“, Theol. Studien und Kritiken 5 (1832), p. 735-768.

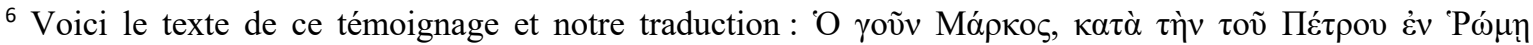

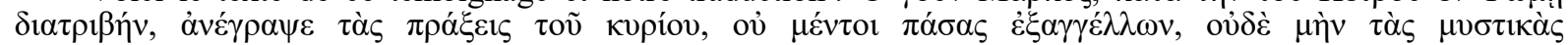

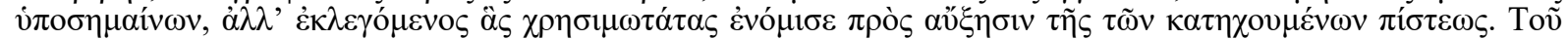

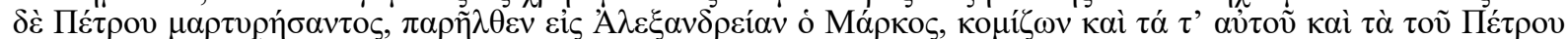

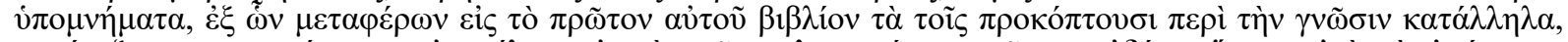

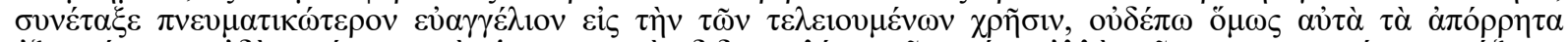

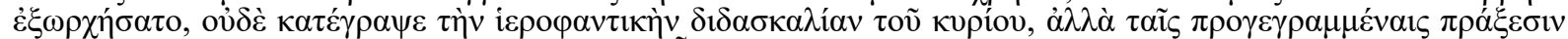

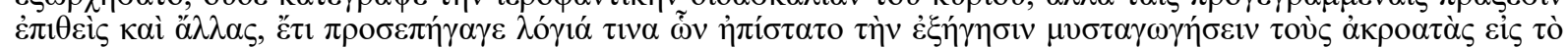

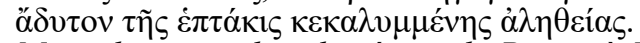

Marc donc, pendant le séjour de Pierre à Rome, rédigea les actes du Seigneur, sans cependant les divulguer tous, ni non plus en indiquer les mystiques, mais choisissant ceux qu'il pensa les plus utiles à l'approfondissement de la foi des catéchumènes. Quand Pierre eut rendu témoignage, Marc se rendit à Alexandrie, emmenant avec ses affaires les mémoires de Pierre à partir desquels, transférant dans son premier livre ce qui convient à ceux qui progressent dans la connaissance, il composa un évangile plus spirituel à l'usage de ceux qui parviennent en fin d'étude, mais il ne révéla pas encore les données les plus secrètes ni ne mit par écrit l'enseignement du Seigneur dévoilant les choses sacrées, mais aux actes déjà écrits en ayant ajouté aussi d'autres, il introduisit encore des paroles brèves dont il savait que l'explication conduirait aux mystères les auditeurs, jusqu'au sanctuaire de la vérité sept fois voilée.
} 
niveau supérieur de formation; (3) et 5 épisodes contenant des paroles de Jésus et ayant également un parallèle seulement dans Matthieu correspondent à un deuxième complément réservé à quelques responsables. Voici ces épisodes :

\section{La catéchèse romaine}
1. Mc 1,21-39
2. Mc 3,7-19
3. Mc 3,31-35
4. Mc 4,21-25
5. Mc 4,35-41
6. Mc $5,1-20$
7. Mc 5,21-43
8. Mc 6,1-6
9. Mc $6,7-13$
10. Mc 9,38-50
11. Mc 12,41-44
12. Mc 14,3-9

\author{
(Lc 4,31-44) \\ (Lc 6,12-19) \\ (Lc 8,19-21) \\ (Lc 8,16-18) \\ (Lc 8,22-25) \\ (Lc 8,26-39) \\ (Lc 8,40-56) \\ cf. Lc 4,16-30 \\ (Lc 9,1-6) \\ cf. Lc 9,49-62 \\ (Lc 21,1-4) \\ cf. Lc 7,36-50
}

\author{
Jésus à Capharnaüm \\ L'appel des Douze \\ Relégation de la famille \\ Trois paroles (lampe, secret rétribution finale) \\ La tempête apaisée \\ Le démoniaque exorcisé à Gérasa \\ Deux femmes guéries au retour \\ Jésus dans sa patrie (réécrit dans Luc) \\ L'envoi en mission \\ Réponse à Jean (réécrit dans Luc) \\ L'aumône de la veuve \\ L'onction de Béthanie (réécrit dans Luc)
}

\section{Le complément narratif}
1. Mc $6,45-56$
2. Mc 7,1-23
3. Mc 7,24-30
4. Mc $8,1-10$
5. Mc 8,11-21

\author{
(Mt 14,22-36) \\ (Mt 15,1-20) \\ (Mt 15,21-31) \\ (Mt 15,32-39) \\ (Mt 16,1-12)
}

\begin{abstract}
Jésus marche sur l'eau
Controverse sur la loi rituelle

La Syro-phénicienne exaucée

Le pain multiplié pour 4000

Rejet des enseignements juifs

P1 L’homme fort ; P2 Le péché impardonnable P3 La semence autonome ; P4 La graine de moutarde P5 Servir et non être servi (parole centrale) P6 Le salut par la foi à déplacer des montagnes P7 Le salut par l'amour, sens de la Loi
\end{abstract}

\section{Le complément de paroles (P)}
1. Mc 3,20-30
2. Mc 4,26-34
3. Mc $10,35-45$
4. Mc 11,12-14.20-26
5. Mc 12,28-34
(Mt 12,22-32)
(Mt 13,24-35)
(Mt 20,20-28)
(Mt 21,18-22)
(Mt 22,34-40)

On ne voit pas encore, sur une simple liste de références et de titres d'épisodes, ce qui fait le lien entre ceux d'une même série. Repérons d'abord les métaphores; nous en viendrons ensuite aux variantes qui donnent accès à un deuxième sens.

\section{Les métaphores d'accès au deuxième sens}

L'exégèse occidentale a perdu de vue la présence d'obstacles dont le récit biblique est chargé et qui ont pour rôle de donner accès à un deuxième sens oublié. Or, il faut accéder à ce deuxième sens pour comprendre la nature du Prôto-Marc. Origène en parlait ainsi : « La sagesse divine a fait en sorte de produire (dans le récit...) des impossibilités et des discordances ; il faut que la rupture dans la narration arrête de lecteur, afin de lui refuser le chemin de cette signification vulgaire [le sens apparent] ... de nous repousser pour nous ramener au début de l'autre voie [le deuxième sens] : ainsi peut s'ouvrir, par l'entrée d'un étroit sentier débouchant sur un chemin plus noble et plus élevé, l'espace immense de la science divine ${ }^{7}$. » Et au $13^{\mathrm{e}}$ siècle, Thomas d'Aquin défendait encore son existence, en distinguant le sens littéral et le sensus rerum ou « sens historique $»^{8}$.

\footnotetext{
${ }^{7}$ Origène, Traité des principes 4, 2,9, trad. Marguerite Harl, Gilles Dorival, Alain Le Boulluec.

${ }^{8}$ Pierre Grelot, Le langage symbolique dans la Bible, Paris, Cerf, 2001, p. 199.
} 


\section{Le prologue de la catéchèse (1,21-39)}

\section{Capharnaüm (v. 21)}

Dès le début du premier épisode, une difficulté apparaît : Jésus arrive à Capharnaüm qui est, au sens apparent, un village de pêcheurs sur la mer de Galilée ; or, ses habitants reconnaissent spontanément l'autorité de l'enseignement de Jésus, tandis qu'ils contestent celui des scribes du temple. Capharnaüm a manifestement un deuxième sens par lequel le mot désigne un milieu hostile au temple, dans lequel Jésus est reconnu d'emblée comme un chef spirituel légitime.

Par son étymologie, Capharnaüm signifie «village $(\mathrm{kpr})$ de celui qui console $(\mathrm{nhm})$ », c'est-à-dire le rassemblement de ceux qui voient en Jésus le consolateur, en grec $\pi \alpha \rho \alpha ́ k \lambda \eta \tau o \zeta$. Mais, pour arriver à ce deuxième sens, il faut une explication, c'est-à-dire le rapport que Jésus a avec ses disciples, après sa prédication en paraboles. L'étymologie de Capharnaüm introduit un deuxième sens.

\section{Exorcisme et guérison (v. 23-34)}

L'activité de Jésus est d'abord d'enseigner devant ses partisans (v. 21-22); puis elle consiste en deux actions médicales, l'exorcisme (v. 23-28) et la guérison (v. 29-31), qui se multiplient, mais en restant distinctes (v. 32-34). Nouvelle difficulté : l'exorcisme ne fait-il pas partie des guérisons ? Pourquoi maintenir la distinction ? Les lieux où se produisent les premiers miracles sont différents : l'exorcisme est lié à la synagogue, c'est-à-dire l'école pharisienne ; et la guérison a lieu à la «maison de Simon », périphrase qui évoque le temple. Comprenons : l'exorcisme est la transformation de l'enseignement pharisien ; et la guérison, une réforme de celui du temple, professé par les scribes.

Ainsi, s'exprimant d'abord devant un auditoire qui lui est acquis (v. 21-22), Jésus produit un enseignement qui transforme celui des pharisiens (v. 23-28) et réforme celui des scribes du temple (v. 29-31). Enfin, à la porte du monde nouveau (v. 32-34), les deux enseignements font place à celui apporté par Jésus.

\section{Le soir et le petit matin (v. 32 et 35$)$}

Les deux moments de la journée indiqués dans le récit encadrent la nuit : (1) le « soir» (v. 32) est un moment de plénitude, les gens qui ont reçu les deux enseignements du judaïsme se sont rassemblés et bénéficient de la correction salvatrice de Jésus, qui exorcise les uns et guérit les autres ; en somme, le salut est accordé à tout le peuple ; (2) mais au "petit matin » (v. 35), Jésus a disparu et ses disciples, menés par Simon, vont à sa recherche et le retrouvent ; commence alors la prédication pour laquelle Jésus est venu (v. 38). Le «petit matin» fait référence à la résurrection de Jésus, qui provoque elle-même la naissance d'une nouvelle prédication.

En somme, tout cet épisode, qui sert de prologue à la catéchèse romaine, est construit comme une métaphore du ministère de Jésus : les épisodes qui suivent vont donc se passer après son ministère, au temps de la communauté primitive, guidée par Jésus ressuscité, toujours vivant, par conséquent, pour les fidèles. 


\section{Le corps de la catéchèse}

Les dix épisodes suivants opèrent un survol de la première génération chrétienne, en reproduisant le plan du Livre des Douze de la Septante ${ }^{9}$, avec (1) un sommaire de la génération $(3,7-19)$; (2) la fondation de la communauté primitive $(3,31-35 ; 4,21-25)$; (3) la gouvernance de Pierre $(4,35-41 ; 5,1-20 ; 5,21-43)$; (4) la gouvernance de Jacques $(6,1-6 ; 6,7-13$; $9,38-50 ; 12,41-44)$.

1) Dans le sommaire, deux listes de noms propres (dont celle des douze apôtres) résument la première génération sous deux angles différents, comme nous allons le voir.

2) Lors de la fondation de la communauté, la direction est refusée par Jésus à sa famille et confiée aux disciples qui font rédiger la collection de ses paroles comme base de leur prédication.

3) La gouvernance de Pierre est faite de miracles qui expriment la transformation du monde par la parole : dissidence des Hellénistes, conversion de Paul et retournement de l'élection divine en faveur de la foule.

4) La gouvernance de Jacques, enfin, est faite d'allusions à quatre événements distants entre eux de sept ans, de la prise de pouvoir de Jacques (42) à sa mort (63) en passant par la conférence de Jérusalem (49) et le séjour de Paul à Éphèse (56). L'absence de miracles, en contraste avec le temps de Pierre, indique que le monde est resté statique, lors de cette gouvernance.

La catéchèse est ainsi habilement conçue pour s'enchaîner avec le Livre des Douze et faire de Jésus celui qui accomplit la promesse faite en Ml 3,1 et rappelée en Mc 1,2 (qui devait servir de sous-titre à la catéchèse) : " Voici j'envoie mon messager... »

\section{L'épilogue de la catéchèse (14,3-9)}

\section{Béthanie (v. 3)}

La catéchèse romaine se termine par une scène d'hommage à Jésus très particulière : une femme verse en pleurant aux pieds de Jésus un parfum de grand prix (v. 3) que Jésus reçoit comme un hommage mortuaire (v. 8), alors que les disciples sont en distance avec ce geste (v. 5), et Jésus proclame un hommage éternel pour la femme qui a eu ce geste (v. 9). Or, la scène se passe à Béthanie. Qu'est-ce donc que Béthanie? Qu'est-ce que cet hommage éternel proclamé par Jésus?

Béthanie est le nom d'un village de Judée; et, par son étymologie, Béthanie, en hébreu byt 'ny, signifie «maison d'Ananias ». Or, un personnage du nom d'Ananias apparaît dans le récit de la conversion de Paul (Ac 9,10-19), pour imposer les mains à Paul et lui faire recouvrer la vue. Le nom de Béthanie crée ainsi un lien avec Paul; et comme nous sommes à la fin de la catéchèse, Paul n'est plus à Damas, mais à Rome, dernier lieu de son enseignement.

\footnotetext{
${ }^{9}$ Entre Osée au début et Malachie à la fin, la Septante dispose les livrets dans l'ordre suivant: Amos Michée - Joël - Abdias - Jonas - Nahum - Habaquq - Sophonie - Aggée - Zacharie ; et dans cet ordre, les livrets se groupent par $1+2+3+4$, pour évoquer un sommaire $(1)+$ le temps de la guerre $(2)+$ le temps assyrien (3) + les temps de la destruction et de la reconstruction du temple (4).
} 


\section{L'ensevelissement de Jésus (v. 8)}

L'hommage, proclamé mortuaire par Jésus, devient alors une allusion à sa mort rédemptrice enseignée par Paul ; et la femme prosternée est l'image de la communauté de Rome qui a reçu cet enseignement. Autrement dit, l'hommage éternel, en épilogue de la catéchèse, concerne donc la ville de Rome, capitale de l'empire et nouveau centre religieux, au moment où Marc rédige sa catéchèse.

\section{Conclusion}

Ainsi, la catéchèse romaine écrite par Marc en 12 épisodes possède un deuxième sens, voilé par le sens apparent, auquel on accède par l'explication des métaphores destinée à quelques-uns, selon le mode d'enseignement que Jésus utilise, d'après Mc 4,33-34, qui conclut un épisode du complément de paroles de la catéchèse romaine $(4,26-34)$ : l'image est pour la foule (sens apparent) et son explication est réservée aux disciples (deuxième sens).

Dans les deux épisodes extrêmes de la catéchèse, aucune variante n'est venue modifier les points essentiels du récit, et le sens métaphorique, s'il ne s'est pas transmis, reste accessible. Mais il arrive que l'accès au deuxième sens ait été corrigé et qu'il ne subsiste que dans certains manuscrits : tels sont les lieux variants dont nous allons à présent parler, car la Vieille latine traduit un texte grec proche de celui du Codex de Bèze ${ }^{10}$, meilleur témoin de la conservation des accès au deuxième sens de la catéchèse de Marc. Nous allons distinguer deux cas : celui où la Vieille latine a perdu l'accès au deuxième sens; et celui où elle a conservé l'accès, alors qu'il s'est perdu dans la tradition grecque.

\section{Les accès aux deuxième sens perdus par la Vieille latine}

Nous allons prendre deux exemples : dans l'épisode de Mc 3,7-19, le Codex de Bèze a conservé plusieurs clés d'accès au deuxième sens, que la Vieille latine a perdues; et en conclusion d'un épisode du complément narratif (Mc 8,1-10), l'un et l'autre ont perdu la clé d'accès.

\section{Mc 3,7-8 : Le couple de « Jérusalem et l'Idumée »}

Le Codex de Bèze (D) atteste ici une variante unique, qui est propre à son texte grec : même la version latine qu'il contient s'en éloigne, en se conformant à la version courante au $4^{\mathrm{e}}$ siècle, avant la révision qu'en fait Jérôme à Rome, de 382 à 384, qui deviendra la Vulgate des évangiles.

On lit cette liste, dans $\mathrm{D}$, avec la syntaxe suivante :

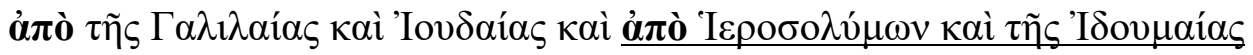

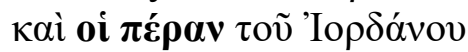

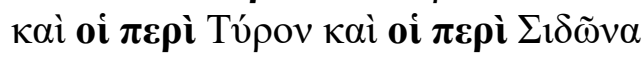

depuis la Galilée et la Judée et depuis Jérusalem et l'Idumée

et ceux au-delà du Jourdain

et ceux autour de Tyr et ceux autour de Sidon

\footnotetext{
${ }^{10}$ Rappelons que ce manuscrit, copié vers 400, a été décrit par David C. PARKER (Codex Bezae. An Early Christian Manuscript and its Text, Cambridge, 1992), et nous avons dirigé ensemble un colloque à Lunel (Codex Bezae. Studies from the Lunel Colloquium, June 1994, New Testament Tools and Studies 22, Leiden, Brill, 1996).
} 
Les sept noms propres, lus dans leur succession, donnent une carte de géographie avec au centre Jérusalem, entre la Terre d'Israël (la Galilée et la Judée) et les pays limitrophes, au sud (l'Idumée), à l'est (au-delà du Jourdain) et au nord (Tyr et Sidon) :

Galilée et Judée

Jérusalem

Idumée, Transjordanie, Tyr et Sidon

Mais si l'on tient compte de la syntaxe, dans le Codex de Bèze, le centre se déplace et la proportion s'inverse : (1) la liste commence par deux couples (depuis la Galilée et la Judée / depuis Jérusalem et l'Idumée); (2) puis vient la nouvelle région centrale (au-delà du Jourdain) ; (3) la liste se termine par deux villes séparées par la répétition de la préposition (autour de Tyr / autour de Sidon); (4) enfin, un article d'enclave (traduit par «ceux ») précède la région centrale et les deux dernières villes.

Cette liste possède, en grec comme en latin, un nombre démesuré de variantes : j'ai compté en grec six énoncés différents de celui du Codex de Bèze et dans la Vieille latine, onze en tout, soit presque un par manuscrit. Il n'est pas utile de les rappeler tous, mais la cause de cette variation inhabituelle est, sans doute, le couple insolite formé par «Jérusalem et l'Idumée »; et deux solutions ont été choisies pour l'éliminer : (1) la suppression de l'Idumée $\left(\mathrm{W} ; \alpha^{*} ; 28 f^{13}(124788)\right.$; VL 6$)$; (2) la répétition de la préposition devant l'Idumée $\left(\Theta f^{1}\right.$; B $\alpha^{\mathrm{c}}$; A Byz; VL 234581013141517 19A, vg). Résumons : la Vieille latine a massivement adopté la dissociation du couple Jérusalem - l'Idumée par la répétition de la préposition, y compris dans la version latine du Codex de Bèze; et par exception, un seul manuscrit (VL 6) supprime l'Idumée.

La leçon qui associe Jérusalem et l'Idumée, dans D, n'est pas accidentel, elle introduit un deuxième sens historique : (1) les quatre premiers toponymes font référence à la dissidence des Hellénistes (Ac 6-8), opposant les disciples, venus de Galilée en Judée, et les Hellénistes, partis de Jérusalem vers l'Idumée (Ac 8,26); (2) le toponyme central est la région où a lieu la conversion de Paul, sur le chemin de Damas (Ac 9); (3) et les deux derniers toponymes représentent le double périple de Paul autour de la mer Égée (Ac 16-20). En bref, nous avons un sommaire de la première génération chrétienne, centré sur la conversion de Paul. Et le couple « Jérusalem et l'Idumée », est nécessaire pour envisager ce deuxième sens historique.

Le Codex de Bèze est le seul manuscrit grec conservant la leçon de cette liste qui mène à une rédaction à deux niveaux de sens; et la Vieille latine en atteste une révision. Cette leçon de D est celle de la rédaction finale des évangiles, faite vers 120 à Smyrne ${ }^{11}$, tandis que les autres manuscrits attestent un texte qui a été ensuite révisé, pour être plus adapté à la lecture liturgique qui devient son usage principal. Et la Vieille latine est dans ce cas.

\section{Mc 3,16-19: La liste des Douze apôtres}

La liste des toponymes propose une vision paulinienne de la première génération, mais une autre liste, celle des Douze apôtres $(3,16-19)$, apporte sur la même période un point de vue différent.

11 C.-B. Amphoux (éd.), Manuel de critique textuelle du Nouveau Testament, Bruxelles, Safran, 2014, p. 274-279. 
La controverse centrale sur les paroles

L'exégèse a déjà repéré que cette liste n'énumère pas les premiers chefs de l'Église, « mais [les apôtres] anticipent sur l'ensemble de l'Église dans sa mission et sa conduite spécifiques », écrit avec un certain embarras Simon Légasse ${ }^{12}$. La liste s'organise en trois groupes successifs de quatre noms dont seul le premier est à la même place, dans les quatre listes du Nouveau Testament (Mt, Mc, Lc, Ac) : Pierre, pour le groupe qui est celui de la première gouvernance communautaire; Philippe, pour le groupe central qui réunit les protagonistes de la controverse sur les paroles de Jésus, en particulier Matthieu et Thomas; et Jacques d'Alphée, pour le groupe qui correspond à la deuxième gouvernance communautaire.

En somme, la liste des apôtres insère entre les deux gouvernances de la communauté primitive le débat sur le sens des paroles de Jésus. C'est une vision plus pétrinienne de la génération : le contenu des paroles est au cœur du conflit avec les Hellénistes ; et cela nous amène à nous interroger sur le nom de Lebbée, donné au dixième apôtre de $\mathrm{Mt}$ et $\mathrm{Mc}$ dans le Codex de Bèze et dans la Vieille latine, les autres manuscrits grecs et la Vulgate lui préférant Thaddée.

\section{Lebbée}

Il faut voir dans Lebbée un mot savant, tandis que Thaddée est le nom d'un personnage dans une légende syriaque du $3^{\mathrm{e}}$ siècle, celle du roi Abgar d'Édesse, reprise par Eusèbe de Césarée (Hist. eccl. 1, 13): Thaddée s'ajoute (Mt) ou se substitue (Mc) à Lebbée, qui a comme correspondant le onzième apôtre des listes de Lc et Ac, « Judas de Jacques ». En voici notre explication : "Lebbée » est un surnom forgé avec les consonnes de Babel $(b-b-l)$ lues à l'envers $(l-b-b)$; or Babel, dans l'épisode de la Tour de Babel (Gn 11), est le signe de la dispersion des hommes ; son inverse serait donc un signe de réunion, mais avec une connotation négative, à cause de Babel. Notre hypothèse est que Lebbée est le surnom d'un personnage qui a fait le choix de la réunion du masculin et du féminin, au sens que lui donne la dissidence des Hellénistes et que l'on trouve dans l'EvTh (22) $)^{13}$, dont l'auteur, qui se nomme lui-même « Judas Thomas », est peut-être aussi Judas de Jacques, dit « Lebbée ».

La Vieille latine atteste bien majoritairement «Lebbée » dans Marc (VL 3 ? 4581314 17), mais avec une certaine confusion : VL 2 a l'absence de ce nom et VL 61015 19A ont Thaddée, qui est la leçon de la Vulgate. Dans VL 2 et 6, le nom supplémentaire de " Judas » figure dans le deuxième groupe, juste avant Matthieu : il indique par sa présence que Lebbée n'est plus identifié comme le surnom du frère de Jésus portant le nom de Judas, dit Jude en français. Du moins la Vieille latine conserve-t-elle, avec Lebbée, un mot savant, mais le lien avec le deuxième sens de la liste des apôtres est perdu.

\section{Iscarioth}

La même observation s'applique au surnom du douzième apôtre, Judas "Scarioth » ou " Iscarioth » : le Codex de Bèze est le seul manuscrit grec attestant « Scarioth », tandis que toute la Vieille latine et la Vulgate la forme sans le I- initial de " Iscarioth ou Iscariote », qui s'est généralisé en grec. Que signifie cette double forme du surnom de Judas, te traître de la passion? La forme «Iscarioth » est la transcription de l'hébreu 'ysh qrywt, « homme (des)

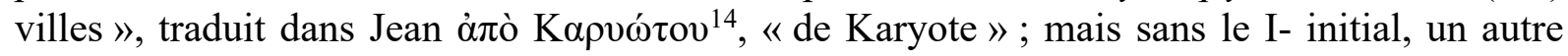

\footnotetext{
12 Simon LÉgAsse, L'évangile de Marc, Lectio divina, Commentaires 5, Paris, Cerf, 1997, p. 235.

${ }^{13} \mathrm{La}$ « réunion » est attestée comme parole de Jésus, dans 2 Clem. 12,2, et expliquée dans le sens de relation fraternelle ; mais avec les Hellénistes, elle a pris un sens philosophique qui l'éloigne de son sens premier.

${ }^{14} \mathrm{Jn} 13,2.26$ et 14,22 , dans $\mathrm{D} ; 6,71$, dans une révision antiochienne $\left(\Theta f^{13}\right)$ et le Sinaïticus $\left(\alpha^{*}\right)$.
} 
substrat hébreu vient s'ajouter, celui de plusieurs verbes, dont shqr, «mentir, trahir, tromper $^{15}$ »; le personnage est donc à la fois « traître » et « prédicateur urbain », double visage qui évoque Paul, avant et après sa conversion, qui prend de l'importance au temps de la gouvernance de Jacques.

La Vieille latine et la Vulgate ont la forme primitive, sans le I- initial du surnom; mais la double étymologie de l'hébreu et la référence à Paul ne sont plus perçues : le rapport envisagé est désormais établi avec le latin sicarius, " sicaire » (tueur à gages), nom donné aux membres d'un courant juif violent, hostile à Rome. Et cette fausse étymologie s'est substituée à l'autre.

\section{Mc 8,10 : Dalmanoutha}

En revanche, ni dans le Codex de Bèze ni dans la Vieille latine on ne trouve Dalmanoutha, à la fin de la multiplication du pain pour 4000 personnes (Mc 8,1-10), qui fait partie de la séquence de Mc 6,45-8,27 correspondant au complément narratif de la catéchèse romaine.

Dalmanoutha reste inexpliqué comme nom de lieu ${ }^{16}$; mais ce mot variant ne s'explique pas à partir des autres leçons, c'est assurément la variante-source. Or, elle ne se trouve, cette fois, ni dans le Codex de Bèze ni dans la Vieille latine. D a une double leçon : $\mu \varepsilon \lambda \varepsilon \gamma \alpha \delta \alpha$ de première main $\left(\mathrm{D}^{*}\right)$, corrigé et traduit par $\mu \alpha \gamma \varepsilon \delta \alpha\left(\mathrm{D}^{\mathrm{c}}\right) /$ magidan (VL 5), qui transcrivent le nom de lieu Meguiddo; et la Vieille latine a aussi mageda(n), sauf VL 10 et 13 qui ont la leçon de la Vulgate (Dalmanoutha).

Dalmanoutha s'explique comme un code numérique qui se calcule en additionnant la valeur de ses consonnes. Selon le code septal en fonction au temple de Jérusalem et découvert par Bernard Barc ${ }^{17}$, Dalmanoutha a une valeur de $24(4+5+6+7+2)$, la dernière consonne étant séparée des autres par un son /o/, ce qui donne le couple de nombres 22 / 24. Or, cette valeur est aussi celle de Boanergès $(3,17)$, dont la traduction par « fils du tonnerre » renvoie à un substrat hébreu bn r'sh, où le gamma transcrit un aïn et non un guimel, ce qui donne 24 (2 $+7+6+2+7)$, la première consonne étant isolée des autres par un son /o/, d'où le même couple de nombres 22 / 24. Ce nombre double évoque, dans la culture du temple, la clôture de l'Écriture, autrement dit l'ajout d'un livre ultime qui viendra clore le canon scripturaire, compté en 22 ou 24 livres. Mais les contextes des deux mots sont différents : Boanergès qualifie deux apôtres, Jacques et Jean, sans indiquer l'aval de Jésus ; tandis que Dalmanoutha est le «lieu » où Jésus emmène ses disciples. Autrement dit, la clôture du canon par les « Boanergès » n'est pas légitimée par Jésus, à la différence de celle qu'indique Dalmanoutha, qui est donc une nouvelle proposition de clôture du canon.

Cette explication permet de rendre compte de l'abandon, dans le Codex de Bèze, de la leçon «Dalmanoutha » et de son remplacement par «melegada»: Dalmanoutha signifiait l'ajout au canon d'un livre ultime ; mais, lors de la rédaction finale des quatre évangiles, il est question de mettre en place une nouvelle Écriture sacrée qui s'ajoute à la Bible juive : « melegada » se substitue à « dalmanoutha » avec la valeur $18(6+5+3+4)$ qui est celle de la Loi (twrh: $1+6+6+5$ ). Et «melegada », devenu rapidement incompréhensible, est corrigé en «mageda» dans la Vieille latine ainsi que lors de la relecture du texte grec du Codex de Bèze.

\footnotetext{
${ }^{15}$ Les autres verbes sont : (1) shkr, « s'enivrer » (cf. EvTh 13) ; (2) skr, « corrompre par de l'argent ».

16 B. HJERL-HANSEN, «Dalmanoutha (Mc 8,10). Énigme géographique et linguistique dans l'évangile de Marc », Revue biblique 53 (1946), p. 372-384.

${ }^{17}$ Bernard BARC, Les arpenteurs du temps, Histoire du texte biblique 5, Lausanne, Le Zèbre, 2000.
} 
En somme, la leçon « Dalmanoutha » a été abandonnée, quand sa signification codée était encore perçue, lors de la rédaction finale des évangiles, qui la remplace par «melegada» (D*), bientôt corrigée en un nom de lieu, « Mageda » (= Meguiddo) (D 28 565, VL 13456 814 15), ou « Magdala » $\left(\Theta f^{1} f^{13}\right)$; puis « Dalmanoutha » est rétabli en grec, dans le texte alexandrin $(\alpha \mathrm{B})$ et le texte byzantin (A Byz), comme s'il s'agissait d'un nom de lieu. On a là un exemple exceptionnel d'une leçon primitive (Dalmanoutha) corrigée lors de la rédaction finale (melegada), puis transformée en toponyme (Mageda, Magdala), avant d'être rétablie dans sa forme primitive comme s'il s'agissait d'un toponyme. Au passage, la forme primitive a été écornée sous la forme « Dalmounaï» $(\mathrm{W})$.

\section{Mc 12,41 : un homéotéleute dans D}

Le Codex de Bèze (D) est le meilleur témoin de la rédaction finale des évangiles, qui corrige parfois les sources utilisées ; mais la copie n'est pas sans faute. Au début de l'épisode de

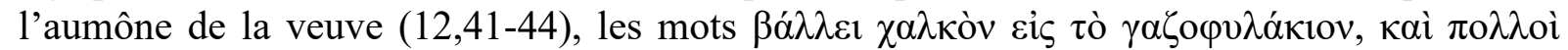

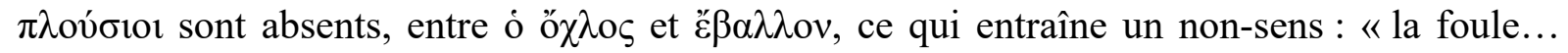
jetaient beaucoup (d'argent) », au lieu de « la foule jette [de l'argent dans le trésor, et beaucoup de riches] (en) jetaient beaucoup ». La Vieille latine ignore cette absence, confirmant qu'il s'agit d'une faute, au moment de la copie du texte grec ; seul, le latin du Codex de Bèze (VL 5) ignore les mots absents du grec et traduit : " la foule mettaient de l'argent », corrigeant ainsi le « beaucoup » du grec.

On voit, dans cet exemple exceptionnel, que la Vieille latine dépend du modèle du Codex

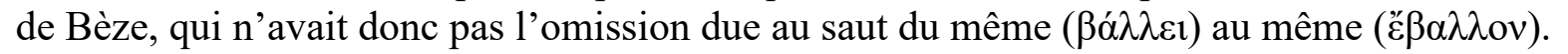

\section{Les accès au deuxième sens conservés par la Vieille latine}

Mais venons-en aux variantes de la Vieille latine qui conservent la leçon primitive, en accord avec le Codex de Bèze. Elles se trouvent à la fois dans le milieu de la catéchèse (Mc 5) et dans le complément narratif (Mc 6,45-8,26).

\section{Les variantes de la catéchèse romaine}

\section{Mc 5,1 : au pays des Géraséniens}

Après la tempête apaisée (Mc 4,35-41), Jésus arrive « au pays des... », avec une variante sur le nom des habitants du lieu: (1) ce sont des Géraséniens, dans D, les deux bibles grecques du $4^{\mathrm{e}}$ siècle $\left(\alpha^{*} \mathrm{~B}\right)$, toute la Vieille latine, la Vulgate et la version sahidique ; (2) mais on a les Gergéséniens dans une révision antiochienne du $3^{\mathrm{e}}$ siècle $\left(\Theta f^{1} 28565700\right)$, une partie du texte alexandrin $\left(\alpha^{\mathrm{c}} \mathrm{L} \Delta 335798921241\right)$, la Vieille syriaque $\left(\mathrm{sy}^{\mathrm{s}}\right)$ et la version bohaïrique, mot désignant une halte de bédouins située sur la rive de la mer; (3) et les Gadaréniens dans le texte byzantin et la Pechitta, par référence à la ville de Gadara, à quelque distance de la mer de Galilée, mais plus proche que Gérasa.

La variante-source est clairement Géraséniens, dont les corrections montrent que la leçon a fait difficulté, car Gérasa est très éloignée de la mer ; les réviseurs ont donc cherché une solution pour diminuer cette distance problématique.

Les mêmes lieux variants existent en Mt 8,28 et Lc 8,26, et le Codex de Bèze (d'après le latin, pour Mt, car le grec est lacuneux) et la Vieille latine ont Géraséniens dans les deux cas ; mais les bibles du $4^{\mathrm{e}}$ siècle ( $\left.\alpha^{*} \mathrm{~B}\right)$ ont Gadaréniens dans Mt, qui est la leçon éditée et traduite aujourd'hui. 
Comment donc expliquer la leçon Géraséniens, qui est primitive dans les trois évangiles? La grande distance entre Gérasa et la mer de Galilée suffit à justifier les corrections.

La situation de Gérasa en fait une étape incontournable sur le chemin de Damas, depuis Jérusalem, autrement dit elle introduit un deuxième sens qui consiste à lire l'épisode comme une métaphore de la conversion de Paul, sur le chemin de Damas. Mais le deuxième sens exige une explication, il ne convient pas à la lecture liturgique, il est donc rapidement abandonné et donne lieu alors à des corrections. On note alors que la Vieille latine est ici témoin comme le Codex de Bèze de la leçon primitive, maintenue dans la rédaction finale des trois synoptiques, puis diversement abandonnée ou maintenue.

\section{Mc 5,22 : absence du nom de Jä̈rus}

Dans l'épisode suivant (Mc 5,21-43), Jésus est interpellé par un "chef de synagogue » dont le nom n'est pas donné dans le Codex de Bèze (D) et une partie de la Vieille latine (VL 2 $\left.3581719 A^{*}\right)$, tandis qu'il figure dans les autres témoins grecs, l'autre partie de la Vieille latine (VL $4610131519 \mathrm{~A}^{\mathrm{c}}$ ) et la Vulgate. Pourquoi cette absence et quelle importance a-telle?

Le nom de Jaïrus est bibliquement marqué, depuis le livre d'Esther, comme faisant référence aux pharisiens, il s'accorde donc avec le sens que l'on prête au mot à $\rho \chi 1 \sigma v v \alpha ́ \gamma \omega \gamma o s$, traduit « chef de synagogue ». Mais la nature du miracle qui suit, une « guérison », suggère un rattachement au temple plutôt qu'à la synagogue, selon le code que nous avons lu dans le prologue (Mc 1,21-39); et l'absence de «Jaïrus » dans le Codex de Bèze semble être la leçon

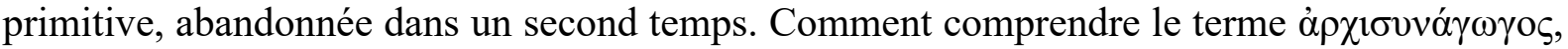
dans ce cas? Existe-t-il au temple une telle fonction ? Le dictionnaire de Bauer (éd. anglaise, Chicago $2000^{3}$ ) donne comme équivalent hébreu $r$ 'sh hknst, " tête de la Grande assemblée », fonction qu'exerce le grand-prêtre en exercice. Il faudrait donc traduire, dans la leçon primitive, "président du Sanhédrin », le mot ayant pris plus tard le sens de « chef de synagogue », et le nom de Jaïrus établit alors un lien avec les pharisiens.

La Vieille latine confirme, d'abord, l'absence de Jaïrus, attestée dans le Codex de Bèze, puis corrige cette absence, et cette correction sera reprise dans la Vulgate.

\section{Mc 5,41 : l’hébreu « tabitha » au lieu de «talitha »}

A la fin du même épisode, Jésus prononce deux mots en langue sémitique généralement lus « talitha koumi », le premier mot étant araméen avec le sens de "jeune fille » et le second, commun à l'hébreu et à l'araméen, est une forme féminine de l'impératif du verbe qwm, " (se) lever ». Mais, dans le Codex de Bèze et la Vieille latine (sauf VL 1013 [et 14 lacuneux]), le premier mot est «tabitha » ou «thabita» («tabea acultha», dans VL 2), qui s'éclaire de la traduction par «jeune fille, je te le dis» et non «jeune fille» seulement : nous y voyons la transcription de l'hébreu tsby 't, " gazelle (= belle jeune fille), toi », mot repris comme nom propre d'une jeune fille, en Ac 9,36. Les passages parallèles de Mt $(9,25)$ et Lc $(8,54)$ n'ont pas les mots sémitiques, et Lc traduit « enfant, lève-toi ».

La reprise du mot « tabitha » dans les Actes explicite le lien qui existe entre les épisodes de Mc 5,21-43 et Ac 9,32-43. Dans Mc, Jésus accompagne un notable (le grand-prêtre ?) pour aller guérir sa fille, mais en chemin, une femme de la foule obtient la première la guérison de son mal; et Jésus arrive à la maison du notable, il guérit sa fille en prononçant le nom de « Tabitha ». Dans Ac, Pierre guérit Tabitha, une Juive sympathisante, mais il a d'abord guéri un malade du nom d'Énée, nom romain par excellence. Dans les deux récits, «tabitha » 
s'applique à celle qui reçoit la guérison en deuxième position, la première profitant à une femme de la foule $(\mathrm{Mc})$ ou un Romain $(\mathrm{Ac})$. De plus, dans la catéchèse romaine,=, l'épisode fait partie de la gouvernance de Pierre. Il est clair que le rédacteur des Actes connaissait l'épisode de Marc avec « tabitha » et non « talitha ».

Dans ces deux livres, l'ordre des guérisons évoque le retournement de la préséance, de l'élection divine, en faveur de la foule contre l'élite sociale (Mc) ou du monde romain contre le peuple juif (Ac), nouvel auditoire confirmé, quand Pierre constate que « le don de l'esprit saint s'est répandu sur les nations » (Ac 10,44-45) ; c'est le grand changement qui se produit au temps de la gouvernance de Pierre, après les deux événements de la dissidence des Hellénistes (Mc 4,35-41 / Ac 6-8) et de la conversion de Paul (Mc 5,1-20 / Ac 9,1-30). Et nous pouvons dater le changement du milieu des années 30, d'après Gal 1,18-19: Paul, trois ans après sa conversion, monte à Jérusalem et rencontre séparément Pierre (v. 18) et Jacques (v. 19) ; sa conversion a eu lieu en 32, soit dix-sept ans avant la conférence de 49, dirigée par Jacques (Ac 15 / Gal 2,1-10); c'est donc en 35 que Paul monte à Jérusalem et suscite le changement qui se produit : Pierre a commencé à prêcher en araméen, comme le faisait Jésus, qui s'adressait ainsi spécifiquement au peuple juif ; mais après la dissidence des Hellénistes et le début de leur enseignement en grec sur les paroles de Jésus, dont témoigne Apollos (Ac 18,24-25), il devient urgent à Jérusalem que l'évangile soit désormais prêché en grec. Paul va donc parler à Pierre en ce sens, et il rencontre Jacques pour la traduction de la collection de paroles en grec, traduction par Jacques confirmée par le Pseudo-Athanase (Synopse de la sainte Écriture, $\S 76)$, dont le propos est cité par Richard Simon ${ }^{18}$ et largement oublié depuis lors.

Ainsi, contre la plus grande partie de la tradition textuelle, la Vieille latine est encore témoin de la leçon primitive «tabitha » remplacée par l'araméen « talitha » dans le texte transmis de Marc. Pour la catéchèse romaine tout entière, grâce à ses leçons conservatrices, on retrouve en traduction le texte de la révision finale des évangiles attestée par le Codex de Bèze, avant l'adaptation du texte grec à la lecture liturgique, à partir du dernier quart du $2^{\mathrm{e}}$ siècle.

\section{Les variantes du complément narratif}

\section{Mc 6,45 : le participe de la résurrection}

Venons-en à la séquence narrative représentant le complément narratif ajouté par Marc à la catéchèse romaine, soit Mc 6,45-8,26. Plusieurs variantes décisives s'y trouvent également, montrant l'accord de la Vieille latine avec les leçons particulières du Codex de Bèze.

L'épisode de la marche de Jésus sur l'eau (Mc 6,45-56 / Mt 14,22-36) prend place au début de cette séquence narrative, qui est ainsi introduite par un miracle stupéfiant. Dans la rédaction finale de Marc, l'épisode vient juste après la multiplication des pains pour une foule de 5000 personnes; et la séquence narrative contient une deuxième multiplication des pains, pour 4000 personnes, cette fois. Ces nombres ont un sens symbolique : " mille » est l'image d'une foule ou d'un peuple, en tant que grand nombre ; et « 5 » et « 4 » s'opposent en ce que le premier est lié à l'alliance, exprimée par le «pain », par référence à $\mathrm{Gn} 18$, la rencontre à Mamré, où Abraham partage le pain avec les trois anges de Dieu; et le second est l'image de

\footnotetext{
${ }^{18}$ Richard SIMON, Histoire critique du texte du Nouveau Testament, Rotterdam, 1689, p. 94
} 
l'humanité tout entière, exprimant le succès de la prédication de Pierre au monde romain ${ }^{19}$. Dans ce double contexte, quel est le sens du miracle de la marche sur l'eau?

Jésus apparaît à ses disciples à la fois corporel et immatériel, il est entre ciel et terre : l'image évoque la résurrection. Et un indice décisif de ce deuxième sens du miracle se trouve au début de l'épisode: la présence d'un verbe de résurrection, au début de l'épisode, mais seulement dans le Codex de Bèze et la Vieille latine ${ }^{20}$.

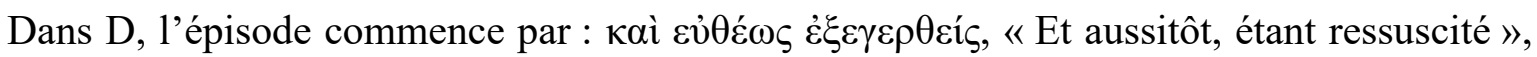
le participe n'étant attesté en grec que dans le Codex de Bèze; mais il est traduit dans la Vieille latine par exsurgens (VL 341314 17) ou par surgens (VL 6 10), à l'exception de VL 1015 qui ont la leçon de la Vulgate, avec l'absence de ce participe. Avec ce participe, le miracle fait allusion aux apparitions du ressuscité à ses disciples, autrement dit, il représente l'événement dont va naître la communauté primitive et s'accorde ainsi avec le témoignage de la lettre de Clément d'Alexandrie, qui associe le premier complément de la catéchèse à des « mémoires » de Pierre. Mais, dans l'usage liturgique de cet épisode, l'allusion à la résurrection laisse la place à l'interprétation de ce miracle comme une manifestation de la divinité de Jésus ; le participe, devenu inutile, est alors retiré du texte. Là encore, la Vieille latine est un témoin précieux de son existence dans la leçon primitive.

\section{Mc 7,24: Tyr sans Sidon}

Les quatre épisodes suivants de la séquence narrative sont disposés en chiasme : le premier A (Mc 7,1-23 / Mt 15,1-20) et le dernier A' (Mc 8,11-21 / Mt 16,1-12) concernent la critique de la loi et des enseignements du judaïsme, celui des pharisiens et celui du temple ; et les deux épisodes centraux B (Mc 7,24-30 / Mt 15,21-31) et B' (Mc 8,1-10 / Mt 15,32-39) ont trait à la prédication qui se tourne vers le monde romain : la Syro-phénicienne est l'image du monde romain en demande d'une religion de salut; et la multiplication des pains marque le succès de cette prédication qui s'ouvre au monde romain. Le chiasme est ainsi en deux parties (Mc 7,1-23 24-30 / 8,1-10 11-21) et, à la fin de chacune, la séquence de Marc insère deux guérisons, celle d'un sourd (7,31-37) et celle d'un aveugle (8,22-26), absentes de Matthieu.

Au début de l'épisode de la Syro-phénicienne, Jésus s'est rendu dans la région de "Tyr », selon le Codex de Bèze et quelques autres manuscrits représentant une première révision (W) ou une révision antiochienne du $3^{\mathrm{e}}$ siècle $(\Theta 28565)$, suivis par deux manuscrits ordinairement alexandrins ( $\mathrm{L} \Delta$ ), tandis que le texte alexandrin ( $\alpha$ B 33) et le texte byzantin (A Byz) ont la leçon «Tyr et Sidon ». La Vieille latine a simplement «Tyr» (VL 34581416 17) et quelques témoins ont la leçon Vulgate, «Tyr et Sidon » (VL 61013 15). Quel est l'enjeu de cette présence ou non de Sidon à côté de Tyr?

Dans la liste des villes et régions que nous avons vue (Mc 3,7-8), Tyr et Sidon, séparés dans la leçon primitive, y représentaient les deux voyages égéens de Paul ; et par référence à cette liste, l'emploi de "Tyr» seul prend sens comme une référence au premier voyage de Paul autour de la mer Égée (Ac 16-18); tandis que le couple « Tyr et Sidon » est une manière de désigner la région située au nord de la Galilée : l'indication est purement géographique, le deuxième sens a disparu. La Vieille latine a conservé en traduction la leçon primitive.

19 Le symbolisme biblique des quatre premiers nombres entiers me semble avoir comme origine l'école pythagoricienne où leur réunion forme la tétraktys. Les nombres impairs $(1,3)$ renvoient au monde céleste $(l e$ ciel, Dieu) et les nombres pairs $(2,4)$, au monde terrestre (la terre, l'homme). Ces correspondances s'appliquent à la structure de la catéchèse romaine.

${ }^{20}$ Notons que cette variante n'est pas signalée, dans l'apparat critique de Nestle-Aland. 


\section{Mc 7,31 : Depuis Tyr par Sidon}

Au début de la guérison du sourd (7,31-37), qui prolonge l'épisode de la Syro-phénicienne, la leçon de $\mathrm{D}$, partagée cette fois par une révision antiochienne du $3^{\mathrm{e}}$ siècle $(\Theta 565700)$ et le texte alexandrin ( $\alpha$ B L $\Delta 33$ 892), mentionne Sidon comme un passage après le départ de Tyr vers la mer de Galilée : le chemin est invraisemblable, car Sidon est au nord de Tyr et la mer de Galilée, au sud-est. L'autre leçon résout la difficulté en coordonnant «Tyr et Sidon », qui désigne à nouveau le pays situé au nord de la Galilée : c'est la leçon des premières révisions $\left(\mathrm{W}, \mathrm{P}^{45}\right.$ [très lacuneux]) et du texte byzantin (A $\left.B y z\right)$. La leçon de $\mathrm{D}$ est celle de la Vieille latine (VL 34568101415 17), à l'exception de VL 13, qui a la leçon byzantine, tandis que la Vulgate conserve la leçon Vieille latine. Et cette leçon, qui fait difficulté, suscite la recherche d'un deuxième sens.

Revenons à la liste des villes et régions de Mc 3,7-8: Sidon renvoyait au deuxième voyage égéen, celui où Paul séjourne à Éphèse (Ac 19-20) et rédige sa lettre aux Galates et la première aux Corinthiens, où prend place la christologie. Au deuxième sens, on comprend, en introduction à la guérison du sourd (7,31-37), que le nouveau miracle doit passer par le deuxième voyage égéen et par conséquent inclut la christologie élaborée à Éphèse.

Quel est donc le sens de cette guérison du sourd, qui doit intégrer la christologie élaborée par Paul ? Le complément narratif, annoncé comme plus confidentiel que la catéchèse, doit retenir l'attention : trois mots vont concentrer le message réservé à un auditoire restreint, selon Clément d'Alexandrie : la surdité, le bégaiement et le mot de Jésus " ephphatha ». (1) La surdité fait jeu de mots avec le verbe hébreu shm', "entendre », sur lequel est formé le nom de Simon $(\mathrm{shm}$ ' $w n)$; (2) le bégaiement du personnage fait référence à la vocation de Moïse $(E x 4,10)$, qui prétexte une difficulté à parler pour ne pas être choisi par Dieu pour conduire le peuple : en somme, il s'agit de définir le profil de celui qui va conduire la communauté des chrétiens, et la nature de l'infirmité nous oriente vers «Simon »; mais il y a deux Simon, le chef des disciples et le cousin de Jésus, fils de Cléopas, qui dirigera la communauté de Jérusalem, après la destruction du temple : lequel des deux Simon aura la légitimité, à la tête des chrétiens de deuxième génération? (3) Le mot de Jésus " ephphatha » est traduit par « ouvre-toi », il transcrit l'hébreu hpth, impératif hitpaël du verbe pth, « ouvrir ». Et, selon le code septal déjà mentionné pour expliquer Dalmanoutha, le mot hébreu a la valeur de $10(5+$ $3+1+1)$, qui est aussi la valeur du radical consonantique de Pierre $(p t r: 3+1+6)$ ou de son équivalent araméen kyp $(4+3+3$, transcrit Céphas, mais aussi Caïphe). Autrement dit, à la génération à venir, le dirigeant légitime sera le successeur de Pierre, qui devra adhérer à la christologie de Paul élaborée lors de son deuxième voyage égéen. Mais cette réponse est masquée, elle est réservée à ceux qui ont accès au deuxième sens.

On peut voir une confirmation de cette analyse dans l'adresse de 2 Pierre, d'un auteur manifestement distinct de celui de 1 Pierre, qui ajoute à son nom «Simon» le surnom de «Pierre": la parenté de cette épître avec celle de Jude nous amène à penser que l'une et l'autre sont écrites par Judas et Simon, nommés comme frères de Jésus, en Mc 6,3 (et Mt 13,55). Qui donc succédera, de fait, à Simon Pierre, dans l'histoire ? Les œuvres de Clément de Rome et d'Ignace d'Antioche font référence à Pierre et à Paul : avec eux, la succession de Pierre a bien été conforme au sens caché de la guérison du sourd.

\section{Mc 8,22 : Béthanie au lieu de Bethsaïda}

La guérison de l'aveugle, qui conclut la séquence narrative (Mc 8,22-26), vient compléter celle du sourd. La connaissance passe par l'audition ou par la vue et la vision. L'audition est le canal dont bénéficient les disciples, dont la connaissance est détaillée, mais éclatée ; l'autre 
canal est celui des visionnaires, qui reçoivent une connaissance globale. Or, Paul, lors de sa conversion, a reçu une telle connaissance : c'est donc à lui que fait allusion cette deuxième guérison ajoutée à la séquence narrative. Mais comment en faire une connaissance réservée, comme la guérison du sourd?

Le lieu où se produit cette guérison est variant : la scène se passe à « Béthanie », dans le Codex de Bèze et la Vieille latine (VL 3 4* 581013 14 17), et à « Bethsaïda », dans tous les autres manuscrits grecs, dans VL $14^{\mathrm{c}} 615$ et dans la Vulgate. Le Codex Bobiensis (VL 1), qui représente le type africain de la Vieille latine que Cyprien avait en mains, vers 250, atteste que la leçon Bethsaïda existe en latin dès le début du $3^{\mathrm{e}}$ siècle ; mais au 4 $4^{\mathrm{e}}$, c'est l'autre leçon qui s'impose. En grec, Béthanie ne se trouve que dans le Codex de Bèze. Quel est l'enjeu de cette variante?

Béthanie est un village de Judée, très éloigné du trajet de Jésus entre Tyr et la mer de Galilée, nouvelle difficulté géographique ; or, dans l'épilogue de la catéchèse (Mc 14,3), nous avons vu que Béthanie, au deuxième sens, faisait allusion à l'école de Paul à Rome : l'aveugle est ainsi également une allusion à Paul, devenu visionnaire lors de sa conversion, puis faisant deux fois le périple de la mer Égée avant de se rendre à Rome, comme le récit de la guérison de l'aveugle indique que le miracle s'opère en deux temps. Béthanie a donc ici le même sens qu'à la fin de la catéchèse ; tandis que Bethsaïda est un port sur la rive nord de la mer de Galilée, côté Décapole, et convient donc géographiquement au contexte de cette guérison. L'élimination du deuxième sens, préparant la destination liturgique des évangiles, rend nécessaire de remplacer Béthanie par un lieu plus proche de l'itinéraire du retour de Tyr, et Bethsaïda s'impose par sa consonance proche de celle de Béthanie.

\section{Conclusion : la découverte du deuxième sens}

La rencontre d'une recherche de la composition littéraire de l'évangile de Marc et de la découverte d'une lettre de Clément d'Alexandrie attestant les conditions de la rédaction du livre écrit par Marc nous a mené à découvrir l'existence du Prôto-Marc, comme une partie de l'évangile comprenant 22 des 66 épisodes. Il s'agit d'une catéchèse intégrée à la rédaction finale de Marc; et l'examen du texte de ses épisodes montre que la rédaction de cette source était savante, ménageant un deuxième sens voilé par le sens apparent et racontant une histoire différente. Grâce au Codex de Bèze, le texte primitif de cette source ne s'est pas perdu, il s'est généralement conservé lors de la rédaction finale de Marc, à l'exception notable de «Dalmanoutha » $(\mathrm{Mc} 8,10)$, à laquelle on peut encore ajouter l'allusion à la multiplication des pains pour $5000(8,19)$, venant d'une autre tradition.

La lecture des évangiles qui s'est transmise n'a pas gardé le souvenir d'un double niveau de sens du texte évangélique, elle est conditionnée par l'usage qui s'est mis en place à la fin $\mathrm{du} 2^{\mathrm{e}}$ siècle et qui s'éloigne de la destination première des évangiles jusqu'à leur rédaction finale, vers 120 à Smyrne, selon un projet conçu par Ignace d'Antioche et attesté par Eusèbe de Césarée ${ }^{21}$. Les évangiles subissent une révolution culturelle au cours du $2^{\mathrm{e}}$ siècle, au terme de laquelle le deuxième sens, réservé et accessible par explication, est évacué au profit du seul sens apparent auquel chacun a directement accès. L'examen de plusieurs lieux variants illustre bien ce changement.

La Vielle latine de Marc, encore attestée par une vingtaine de manuscrits et de nombreuses citations patristiques, puis révisée par Jérôme pour devenir la Vulgate, occupe une place à

${ }^{21}$ Hist. eccl. 3, 36,4. 
part, dans l'histoire du texte de cet évangile. La traduction se fait indépendamment des révisions grecques et sera revue à plusieurs reprises en privilégiant un modèle qui n'est ni alexandrin ni antiochien, mais se conforme à la rédaction finale tout en étant destinée à la lecture liturgique. Il en résulte une version pleine de difficultés pour la compréhension de détail, et Jérôme trouvera une solution en suivant un modèle grec proche du Codex Vaticanus (B), peut-être en utilisant ce manuscrit grec lui-même, qui semble avoir été copié à Rome sur des modèles venant d'Alexandrie, sous l'autorité d'Athanase, alors en exil à Rome ${ }^{22}$. Le témoignage de la Vieille latine confirme l'existence d'un Prôto-Marc intégré à la rédaction finale de cet évangile, dont le texte est encore accessible par le Codex de Bèze et cette première version latine.

\section{BIBLIOGRAPHIE}

Amphoux, C.-B. (éd.), 2014 : Manuel de critique textuelle du Nouveau Testament, Bruxelles, Safran.

AndRIST, P., 2009 : Le manuscrit B de la Bible (Vat. Gr. 1209) (Histoire du texte biblique 7), Lausanne, Le Zèbre.

BARC, B., 2000 : Les arpenteurs du temps (Histoire du texte biblique 5), Lausanne, Le Zèbre.

Grelot, P., 2001 : Le langage symbolique dans la Bible, Paris, Cerf.

HAELEWYCK, J.-C., 2013-2018 : Evangelium secundum Marcum, Vetus latina, Die reste der altlateinischen Bibel, vol. 17, Freiburg-im-Brisgau, Verlag Herder.

HJeRL-HANSEN, B., 1946 : «Dalmanoutha (Mc 8,10). Énigme géographique et linguistique dans l'évangile de Marc », Revue biblique 53, p. 372-384.

KAEstLI, J.-D., 1997 : «Evangile secret de Marc », dans F. Bovon, P. Geoltrain (dir.), Écrits apocryphes chrétiens, tome I (Bibliothèque de la Pléiade 442), Paris, Gallimard, p. 55-69.

LÉGASSE, S., 1997 : L’évangile de Marc (Lectio divina, Commentaires 5), Paris, Cerf.

PARKer, D.C., 1992 : Codex Bezae. An Early Christian Manuscript and its Text, Cambridge,

PArker, D.C., Amphoux, C.-B., 1996 : Codex Bezae. Studies from the Lunel Colloquium, June 1994 (New Testament Tools and Studies 22), Leiden, Brill.

SCHLEIERMACHER, F., 1832 : «Über die Zeugnisse des Papias von unsern beiden ersten Evangelien“, Theol. Studien und Kritiken 5, p. 735-768.

SimON, R., 1689 : Histoire critique du texte du Nouveau Testament, Rotterdam.

SMith, M., 1973 : Clement of Alexandria and a Secret Gospel of Mark, Cambridge, Mass.

\footnotetext{
${ }^{22}$ Patrick ANDRIST, Le manuscrit B de la Bible (Vat. Gr. 1209), Histoire du texte biblique 7, Lausanne, Le Zèbre, 2009.
} 


\title{
RÉSUMÉ
}

En isolant l'ensemble des épisodes disposés dans le même ordre dans les trois synoptiques, on observe que le reliquat s'organise en trois séries narratives correspondant au témoignage récemment découvert de Clément d'Alexandrie, qui décrit ainsi un prôto-Marc. Et pour lire ce premier livre hypothétique, on doit prendre en compte les variantes du Codex de Bèze, que confirme généralement la Vieille latine, récemment éditée pour Marc par Jean-Claude Haelewyck.

\begin{abstract}
By isolating all the episodes arranged in the same order in the three synoptics, we observe that the remainder is organized into three narrative series corresponding to the recently discovered testimony of Clement of Alexandria, who thus describes a proto-Mark. And to read this first hypothetical book, one must take into account the variants of the Codex Bezae, which is generally confirmed by the Old Latin, recently edited for Mark by Jean-Claude Haelewyck.
\end{abstract}

\section{MOTS-CLEFS}

1. Philologie biblique

2. Composition littéraire

3. Histoire du texte

4. Prôto-Marc

5. Vieille latine

\section{KEYWORDS}

1. Biblical philology

2.Literary composition

3.Text history

4. Proto-Mark

5. Old Latin 